\title{
La presencia de Augusto en Hispania y las fechas de la reoganización provincial
}

\author{
Gonzalo Bravo CASTAÑEDA \\ Universidad Complutense de Madrid \\ gbravo@ucm.es
}

\section{RESUMEN}

Las fechas citadas en este estudio remiten a la presencia de Augusto en la Península Ibérica con el fin de reorganizar los territorios y pueblos hispanos tras la conquista romana. Esta nueva organización incluía la división provincial, que probablemente fue tardía y, por supuesto, no se realizó de una sola vez, sino tras varios intentos, tal como puede verse en el Edicto de El Bierzo del año 15 a.C., cuya interpretación debería ser revisada. Se aportan aquí nuevos argumentos que cuestionan a los legati citados en este texto epigráfico como auténticos gobernadores provinciales.

Palabras clave: Historiografía. Fuentes. Viajes de Augusto a Hispania. Bronce de El bierzo. Procónsul. Provincia Transduriana. Legados.

\section{The Augustus' Presence in Spain and the Dates of the Provincial Organization}

\begin{abstract}
The dates cited in this paper refer to the presence of Augustus in the Iberian Peninsula in order to reorganize the territories and Hispanic peoples after the Roman conquest. This new organization included the provincial division, which was probably late and, of course, was not done once, but after several attempts, as you can see in the Edict of the Bierzo (León, Spain) (15 BC), whose interpretation should be reviewed. We bring here new arguments that question the legati cited in this epigraphic text as authentic provincial governors.
\end{abstract}

Key Words: Historiography. Sources. Augustus in Spain. The Bronze from El Bierzo. Proconsul. Transdurian Province. Legati. 
Es obvio que muchos de los problemas históricos no han sido resueltos por la historiografía de forma satisfactoria, de ahí que convenga volver sobre ellos de vez en cuando entendiendo que a menudo en historia las conclusiones de "ayer" suelen ser puntos de partida de "hoy", tanto si refiere a un nueva interpretación de las fuentes como a una revisión de las teorías. Pero la historiografía augustea es, de hecho, inabarcable: cientos de monografías individuales y colectivas se escriben cada año sobre su figura y obra política, administrativa, económica, cultural, ideológica o religiosa. En efecto, resulta difícil hallar en tan copiosa historiografía un aspecto realmente nuevo, es decir, no tratado con anterioridad en monografías ya clásicas, en estudios sobre Augusto y su época, en Congresos sobre la figura de Augusto, en artículos en revistas especializadas o de divulgación o en Catálogos de Exposiciones. ${ }^{1} \mathrm{Y}$, aunque en menor medida, también es ya importante la bibliografía sobre la Hispania de Augusto, de forma que no puede ser obviada. A pesar de ello, hoy nos proponemos "descubrir" algún aspecto velado todavía de esa "inmensa biografía" o al menos aportar nuevos datos y argumentos sobre hechos poco o mal conocidos, que nos ayuden a comprender mejor, entre otras cosas, por qué la figura de Augusto ha dado nombre a todo un siglo (saeculum), la época denominada tradicionalmente como "el siglo de Augusto".

No obstante, la propia historiografía -muchas veces sin ser consciente de ellosuele ayudar a resolver el problema, en cuanto que a la vez que nos permite la crítica de interpretaciones no satisfactorias de fuentes y problemas, nos proporciona también a menudo modelos de análisis que, adecuadamente adaptados a "nuestro objeto" sugieren nuevas vías de solución. Pues bien, desde esta perspectiva trataremos de responder hoy a cuatro importantes cuestiones, no resueltas satisfactoriamente en $\mathrm{mi}$ opinión hasta el momento:

1. Si las fechas tradicionales de la reforma provincial augustea son aplicables al caso hispánico (el año 27 a.C.).

2. Si, en defecto de otras fuentes de información, la presencia de Augusto en Hispania, atestiguada tanto en fuentes literarias como arqueológicas, es un factor clave para fijar las fechas de la reorganización provincial.

3. Si la reforma provincial augustea se llevó a cabo como la describen las fuentes tradicionales conforme a un hipotético reparto "pactado" de provincias entre el Senado y el emperador de una sola vez o la reconstrucción de los hechos permite otras alternativas sobre el procedimiento en que esta se llevaría a cabo.

4. Si los llamados Fasti Hispanienses de época augustea corroboran o no la reconstrucción tradicional.

El segundo elemento de análisis es la cronología, referida a las fechas de la reorganización provincial augustea, distinguiendo en todo caso entre las medidas adoptadas por Augusto respecto a las comunidades existentes de las referidas a la ordenación del territorio y la organización de nuevas entidades administrativas y territoriales (conventus y provincias, especialmente) diseñadas por el princeps para la Península ibérica.

\footnotetext{
1 Por citar sólo algunas obras representativas de la historiografía moderna y reciente: JoNES 1974; SymE 1989; Bowman ET ALII 1996; GaLINSKi 2005; ECK 2003; FrasCheTti 2003; Lillo RedONEt 2009; La RocCA ET ALII 2013.
} 
Respecto a la cronología, la historiografía sigue siendo en exceso tributaria de la documentación literaria, que adscribe la reforma provincial augustea (de todo el Imperio) a un solo año (27 a.C.), ${ }^{2}$ e incluso a un solo lugar (el Senado romano), a un solo momento (la sesión del 16 de enero) y a una sola actitud política (pacto entre emperador y Senado). En general, sin embargo, los historiadores modernos han aceptado esta fecha tomando como referencia a los autores antiguos que hablan sobre ella, especialmente Estrabón, Plinio y Dión Cassio. ${ }^{3}$ Según los datos aportados por estos autores, en el año 27 a.C. la línea del Duero se establecía como límite entre las dos nuevas provincias de Lusitania y Tarraconense, y los "distritos" 4 de Asturia et Gallaecia estarían incluidos en Lusitania, de la que serían separados después. Pero hoy estamos en condiciones de cuestionar esa fecha porque, al menos por lo que se refiere a Hispania, la reforma no pudo realizarse plenamente antes del 19 a.C. (fin de las guerras cántabras) o del 16 (inicio del segundo viaje), quizá no antes del 15 a.C. (Bronce de El Bierzo) ni probablemente antes del 13 a.C. (regreso de Augusto a Roma y acuerdo del Senado para erigir el Ara Pacis en su honor), ${ }^{5}$ aunque al menos una parte de esa reforma ya estaría en vigor en el 12 a.C. ${ }^{6}$

No obstante, la documentación disponible en los Fasti provinciales -salvo excepción (Lusitania)- no refleja estos cambios hasta varios años después, como veremos más adelante. De hecho, se podría pensar que la reorganización no concluyó hasta el 7 a.C. ${ }^{7}$ o incluso el 2 a.C., ${ }^{8}$ cuando parece que se habían consolidado los límites definitivos de la Citerior a expensas de la Lusitania por el norte (Asturia y Gallaecia) y de la Ulterior por el sur (Bética), aunque sigue siendo inexplicable por qué en las Res Gestae divi Augusti, que se supone fueron escritas poco antes del 14 d.C., Augusto afirma que "fundó colonias militares in utraque Hispania", referidas probablemente a la Citerior y la Ulterior y no a las tres nuevas provincias. ${ }^{9}$ En cualquier caso, estas cuestiones demuestran que la reorganización provincial es algo más que un problema de cronología, cualquiera que sea el terminus propuesto para su delimitación; es también y ante todo un proceso entre República e Imperio, con límites difusos y sin aparente solución de continuidad. ${ }^{10} \mathrm{Si}$ el programa imperial no estaba aún cerrado en

2 Aunque el texto de las Res gestae 34.1-3, referido a ese año no dice nada sobre "las provincias" ni de la reforma provincial, sino que habla de la devolución de los poderes al Senado (vid. CANALI 2002, 72-73).

3 Especialmente Estrabón, III 4.20; Plinio, NH 4.118; y Dión Cassio, LIII 12.4-5: el Duero como límite entre Lusitania y Tarraconense: Asturia et Gallaecia incluidas en Lusitania, de la que serán separadas después.

4 Vid infra n. 32.

5 RGdA 12:2 (vid. Canali 2002, 28-29).

6 Según el documento llamado generalmente "Mapa de Agripa", transmitido por Plinio, que registra la medición de 24 regiones del Imperio hacia el 12 a.C. (las referidas a Hispania en $N H$ 4.118: menciona ya la provincia de Lusitania, que incluye los territorios de Asturia y Gallaecia, recién conquistados: Así SANTOS 1985, 58), poco antes de la muerte del yerno de Augusto y su más estrecho colaborador; la descripción de este documento también conocido como Orbis pictus (vid. NicOLET 1988, cap. V) se completa con las informaciones de otros documentos posteriores, editados por RiESE 1878, reimp. 1964; sobre la interpretación de los datos aportados, López BARJA 2000, 37-38.

7 AlFöldy 1969, 207.

8 Vid. ahora Bravo Bosch 2008, 132.

9 RGdA 28:1: Colonias in Africa, Sicilia, Macedonia utraque Hispania (...) militum deduxi; conviene recordar que uterque solo puede referirse a "uno y otro", esto es, a ambos de dos.

10 Salinas $1995,170$. 
estos años, tampoco sería definitiva la división provincial hispánica.

Por eso, el tercer elemento de análisis son los viajes a Hispania por parte de Augusto y sus colaboradores así como los posibles itinerarios. La verdad es que contamos con dos estudios recientes, realmente innovadores y modélicos en su género, que nos evitan analizar muchos de los datos conocidos al respecto, a los que, no obstante, remitiremos a menudo. Nos limitaremos en cambio a glosar algunas de sus importantes conclusiones y a presentar aquí una breve sinopsis de los mismos. El primero, se refiere al primer viaje de Octaviano, en el 45 a.C., ${ }^{11}$ retrasado por haber estado convaleciente "en Roma" de una enfermedad, no pudiendo, por tanto, reunirse con César-como se supone a menudo- en la campaña de Munda contra las tropas de los pompeyanos, y siendo fuente capital para el asunto la biografía sobre Augusto escrita por Nicolás de Damasco ${ }^{12}$ frente al testimonio de otros autores, que dan a entender la participación directa de Octavio en esa batalla. El segundo estudio, referido especialmente al segundo viaje, entre los años 26 y 24 a.C., revisa los datos tradicionales y los actualiza con nuevos epígrafes recientemente descubiertos en Segobriga y otros lugares, para proceder después a una nueva reconstrucción de los hechos. ${ }^{13}$

Tan solo una breve sinopsis de las conclusiones. Como es sabido, la documentación histórica registra al menos tres viajes de Octavio-Augusto a Hispania:

El primero en 45 a.C. para reunirse con César después - y no antes ni durante - la batalla de Munda (en el año 45 a.C.) contra las fuerzas pompeyanas concentradas en el sur peninsular, según el testimonio de la Vida de Augusto de Nicolás de Damasco frente al de Dión Cassio, entre otros. En el verano del 27 a.C. Augusto, probablemente después de la celebración del triumphus de M. Licinio Craso ${ }^{14}$ abandonó Roma en dirección a Galia o Britania, según Dion Cassio, aunque se habría dirigido a Hispania por la situación de inestabilidad en los pueblos del norte peninsular.

Este segundo viaje, entre 26 y 24 a.C., con motivo de las guerras cántabras y astures, es, sin duda, el mejor conocido, aunque la reconstrucción del itinerario sigue siendo confusa, debido sobre todo al hecho de que Augusto, en esta ocasión, parece haberse preocupado más de promocionar ciudades que de organizar las nuevas provincias. ${ }^{15}$ Pero el relato aportado por Dión Cassio (DC) es ciertamente farragoso y merece un mínimo comentario: lo primero que llama la atención es el aparente desorden en que un autor de la talla historiográfica de Dión Cassio describe los hechos sin orden lógico ni cronológico. ${ }^{16}$ Pero si este relato de los hechos se ordenara por años, la secuencia sería:

\footnotetext{
11 Perea 2005.

12 Ver ahora la edición, con traducción, introducción y comentarios de PEREA 2006.

13 Abascal 2006.

14 Halfmann 1986, 157.

15 Sobre todo AbASCAL 2006, 68-70; así se explicaría también las numerosas estatuas erigidas en su honor: NAVARro 2001, 36.

16 Así en DC, HR 53, encontramos: 25.2 (referido al año 25 a.C.): “ Augusto planeaba una expedición a Britania, pero se detuvo (en la Galia) por la revuelta de los salasos y por "la hostilidad de los cántabros y astures"; pero en 22.5 (referido al año 27) DC ya había anunciado que "desde la Galia se dirigió a Hispania y restableció también en ella el orden"; sin embargo, en 25.5 (referido al año 25 a.C.) DC insiste en que "el mismo Augusto dirigió simultáneamente la guerra contra los cántabros y los astures", pero en 25.7 (referido a un año antes: 26 a.C.) informa de que "aquejado por la enfermedad tuvo que retirarse a Tarraco, donde asumió el octavo consulado (a. 26 a.C.) y ejercería el noveno (año 25 a.C.) in absentia e iniciaría el $10^{\circ}$ el $1^{\circ}$ de enero
} 
a. 27: DC, 53.22.5;

a. 26: DC, 53.25.7;

a. 25: DC, 53.25.2 y 25.5.

No obstante, el orden lógico sugiere una ordenación diferente:

25.2 (en Galia);

25.5 (en Hispania, al frente del ejército romano);

25.7 (en Tarraco, enfermo);

22.5 (en Hispania, restableció el orden).

Es decir, el orden de exposición de los hechos por Dión Cassio es confuso:

DC, 53.22 .5 (a. 27);

25.2 (a. 25);

25.5 (a. 25$)$

25.7 (a. 26).

Como en su día ya observó A. H. M. Jones, durante este segundo viaje la labor política de Augusto fue muy intensa entre las comunidades hispánicas, afectando al menos al $20 \%$ de las existentes ${ }^{17}$ entre las que se encontraban : al menos 25 colonias de veteranos, otros tantos municipia y no menos de 50 ciudades de derecho latino, nuevas o promocionadas, cifras que comparadas con las de otras provincias de Occidente durante el mismo período son aun más significativas: en Galia, 9 colonias y 27 ciudades latinas; en África, 6 colonias, 15 municipios y 1 ciudad latina; en Sicilia, 5 colonias, 2 municipios y 3 ciudades latinas. ${ }^{18}$

El tercer viaje, en fin, entre 15 y 13 a.C., destinado principalmente a reorganizar los territorios, una vez finalizado el proceso de conquista por Agripa en 19 a.C., que recibió también el imperium proconsulare desde ese año -15 a.C. y no el 16 a.C.- o verano del 16 a.C., cuando supuestamente Augusto abandonó Roma en dirección a Galia o Hispania, según los testimonios de Veleyo, Suetonio y Dión Cassio. ${ }^{19}$ Pero ahora tenemos constancia epigráfica a través del Edicto de El Bierzo de que de nuevo en febrero del año 15 a.C. Augusto estaba en la Galia (en Narbona).

En definitiva, cambios internos en la vida de las comunidades que se completarían con la modificación del "mapa" provincial hispano entre 15 a.C. (fundación de ciudades en Hispania) ${ }^{20}$ y 13 a.C., si no algunos años después, ${ }^{21}$ como hemos visto, aprovechando la presencia en Hispania de Augusto. Estos cambios habrían quedado reflejados en el llamado "documento de Agripa" antes de su muerte en el año 12 a.C., en el que ya se menciona la nueva provincia de Lusitania, tal como lo transmite Pli-

\footnotetext{
del año 24 a.C., antes de su regreso a Roma pocos meses después: Halfmann 1986, 157: "lo más tarde el 13 de junio del 24 a.C."

17 JONES $1974,125$.

18 Ibid. 125-126.

19 Veleyo, 2,97; Suetonio 23 y DC 54.20; también HaLfMANn 1986, 158.

20 Según Dión Cassio, 54.23.7; y ahora ABASCAL 2006, 70-72.

21 ALFÖLDY 1969, 207 proponía ya como fecha más probable para esta reforma el intervalo "entre el 12 y el 7 a.C.".
} 
nio, que incluyó en principio los territorios (regiones) de Asturia y Gallaecia. ${ }^{22}$

4. En fin, el cuarto elemento de análisis es el procedimiento seguido por Augusto para llevar a cabo la reorganización provincial en Hispania. En primer lugar, hay que tener en cuenta que las "grandes reformas" administrativas -y la de Augusto lo era, pero también la de Diocleciano, casi tres siglos después- no se hicieron de una sola vez sino que se consolidaron a lo largo de un proceso más o menos largo de adaptaciones y ensayos. En este proceso debieron concurrir una serie de elementos necesarios para llevar a cabo la reforma con éxito, entre otros los siguientes: 1 . Una información suficiente sobre la situación real del territorio así como de las comunidades existentes; 2. Desplazamiento del personal de la administración pertinente y capaz de realizarla; 3. Distribución de competencias relativas a gobernador, legados y colaboradores; 4 . Solución de posibles problemas jurídicos o sociales que pudieran plantearse; y 5. Presencia in situ del emperador o de alguno de sus colaboradores directos. En este sentido, es significativo que, si la reconstrucción histórica a partir de los documentos es correcta, se constataría la presencia de Augusto en el escenario hispánico antes, durante y después de la conquista. Probablemente la presencia de Augusto, sin ser necesaria quizás en las negociaciones, impregnaba de autoridad y legalidad las decisiones adoptadas por las autoridades romanas destinadas a Hispania. Parece obligado preguntarse, entonces, como lo hizo Crook, ${ }^{23}$ si la presencia de Augusto en Hispania se podría haber debido a razones militares (acabar la conquista de los pueblos del norte peninsular), políticas (de propaganda o fama ante el pueblo romano) o más bien económicas (control sobre las minas del noroeste hispano). En cualquier caso, la reorganización provincial de estos años, en los que Asturia y Gallaecia pasaron a depender definitivamente de la Citerior ${ }^{24}$ desligándose de Lusitania, completaba además los cambios de estatuto jurídico de buena parte de las comunidades hispánicas. ${ }^{25}$ Por tanto, es razonable pensar que la reorganización provincial no se hizo de una sola vez ni seguramente "desde la cancillería imperial" (de Roma ni de Narbona) ni probablemente in situ, pero la presencia de Augusto en el escenario de los hechos y el conocimiento directo de la situación debieron de ser determinantes para llevarla a cabo y adoptar las medidas pertinentes por parte del emperador y sus consejeros.

Desde esta perspectiva, resulta ilustrativo el último documento que traemos a colación: el Edicto del año 15 a.C., también llamado Bronce de Bembibre, o de El Bierzo e incluso Tessera de Paemeiobriga o Aes Bergidense. ${ }^{26}$ Este documento epigráfico, cuya interpretación es aún muy discutida, nos permite comprender mejor los cambios

22 Plinio 4.118.

23 CROOK 1996, 166, enfatiza la diferencia entre propaganda (victoria y pacificación) y realidad ("largo proceso sangriento y brutal").

24 ALFÖLDY 1969, 224: "ausserdem wurden Asturien und Gallaecien, wo die niederspanischen Legionen standen, der Provinz Hispania Citerior einverleibt”. Según Alföldy, la razón de la inclusión de la zona noroeste hispánica en la provincia Tarraconense debió de ser militar: poner todas las tropas legionarias bajo el mando del legatus Augusti de mayor rango en Hispania, el de la Tarraconense.

25 Especialmente RichardSON 1998, 136.

26 Vid. SÁnchez-Palencia - Mangas 2000; Grau-Hoyas 2001;Alföldy 2001; Costabile-Licandro 2000; Martín - Gómez-PANTOJa 2001. 
introducidos por Augusto en comunidades y territorio con vistas a la nueva reorganización provincial. Pero de dicho documento me interesa hoy comentar solamente dos datos importantes en este sentido: 1. El título de procónsul, que se otorga el propio emperador el año 15 a.C.; 2. La mención de provincia, referida al territorio "transduriano". En cuanto al primero, resulta extraño que Augusto se autotitulara así, en esta fecha, cuando la adopción del título de Imperator en su propia nomenclatura desde el 27 a.C. y el otorgamiento del imperium proconsulare por el Senado en 23 a.C., harían innecesaria esta mención. Además, nótese que no traducimos "en calidad de procónsul" (en ablativo) ni "como si fuera un procónsul", sino justamente "Augusto, procónsul" (en nominativo), lo que podría ser considerado un auténtico "háppax" en la epigrafía augustea, que reclama un mínimo comentario. En efecto, como ha sido justamente observado, se trata del único documento epigráfico que atribuye este título oficial a Augusto. ${ }^{27} \mathrm{Si}$, no obstante, se especifica este, parece deberse a dos razones: una, el interés del emperador en dictaminar "como procónsul", ${ }^{28}$ de la misma forma en que lo habría hecho un procónsul republicano con las atribuciones correspondientes otorgadas por el Senado: delimitar territorios, establecer límites de jurisdicción, conceder privilegios, otorgar la ciudadanía, asignar cargas tributarias a unas comunidades y exonerar a otras y, en definitiva, reorganizar los territorios y modificar el estatuto de las comunidades; dos, los gobernadores, tanto si eran de tipo republicano como si seguían ya el modelo imperial recientemente implantado, sólo tenían competencias jurisdiccionales sobre su provincia/provincia, pero no fuera de ella ni tampoco en al ámbito de las relaciones interprovinciales. ${ }^{29} \mathrm{Y}$ esto nos lleva directamente a la segunda cuestión enunciada arriba: la "provincia transduriana" mencionada en el texto.

En nuestra opinión, expresada por escrito en repetidas ocasiones, ${ }^{30}$ si la anterior argumentación es correcta, resultaría más fácil entender que todavía aquí - en febrero del año 15 a.C. - se hable de provincia transduriana en el sentido republicano del término "provincia" como mandato extraitálico de un magistrado, ${ }^{31}$ y no en el sentido territorial-administrativo de las circunscripciones posteriores de época imperial al mando de un gobernador, ${ }^{32}$ aunque quizás se trate solamente de un "ensayo" imperial que, de momento, no se consolidó. Desde esta perspectiva, la mención del legado Lucio Sestio Quirinal en el edicto no debería entenderse quizás como la de un "gobernador provincial" con título de legatus (Augusti), sino como referida a un representante ocasional del emperador, enviado a esa zona con un mandato temporal

27 EcK 2003, 58.

28 No et pro consule (así la transcripción y comentario de ALFöLDY 2001, 17 y 19) sino et proconsul o, si se prefiere, actuando como tal en sustitución de los procónsules, en un sentido similar al propuesto por SyME 1970, 85 atribuyendo a Augusto el control sobre toda Hispania desde el año 27 a.C., cuando desaparecieron los procónsules o los existentes fueron solamente legati personales del propio princeps.

29 Así Millar 1966, 157, como rasgo común a todo tipo de gobernadores provinciales con independencia de su rango.

30 Bravo 2001, 50-51; Bravo 2007, 102-105; Bravo 2011, 142-143.

31 BRAVO 2001, 51.

32 Así expresamente LóPEz BARJA 2000, 32: "Creo que nos hallamos ante una auténtica provincia territorial, que había sido conformada como tal pocos años antes y que, en el fondo, sólo se diferencia de otras como la Tarraconense o la Bética por su corta vida". 
y con una misión especial, que el propio documento especifica: informar al emperador de la actitud hacia los romanos (de colaboración o de resistencia) de las diversas comunidades del noroeste hispano (área "transduriana"), misión a la que -prosigue el documento- fueron enviados varios legados: "de todos mis legados". Alföldy hablaba de la "provincia transduriana" como una especie de "subdistrito provincial". ${ }^{33}$ Pero este uso del plural posesivo referido a "legados" (cognovi ex omnibus legatis meis) no debe ser casual y, por tanto, no tendría sentido en una "provincia" recién constituida ni ajena al emperador. Resulta equívoco asimismo el uso del posesivo en el texto (meis) ${ }^{34}$ lo que induciría a pensar ya en auténticos legati Augusti pro praetore (es decir, gobernadores provinciales de época imperial) cuando en realidad debe tratarse de uno de los legati ayudantes del legatus Augusti correspondiente o de legados militares al mando de las respectivas legiones, que en el caso de la nueva provincia Tarraconense, con un gobernador de rango consular, serían tres los asignados a su mandato: según Estrabón, uno dirigía una legión y controlaba la zona cántabro-pirenaica; otro, sin mando legionario, administraba la zona ya pacificada del Valle del Ebro; y un tercero, al mando de dos legiones, controlaba la zona asturgalaica. ${ }^{35} \mathrm{~A}$ este último es al que presumiblemente se refiere el edicto del año 15 a.C. cuando se alude al "mando" en el "área transduriana". Pero hay además una razón jurídica, que apenas ha sido tenida en cuenta o poco valorada por la historiografía, en cuanto que sólo una autoridad superior - y no los simples gobernadores provinciales - tenía atribuida la competencia para tomar medidas que afectaran directamente a los intereses de ciudadanos romanos o latinos ${ }^{36}$ como los que ya había en Hispania, por lo que Estrabón afirmaba que "las ciudades latinas escapaban a las órdenes de los gobernadores" ${ }^{37} \mathrm{y}$, aunque cayeran dentro de su jurisdicción, no tenían la autoridad suficiente para intervenir fuera de los límites de su "provincia" ni probablemente en cuestiones que atañeran a sus privilegios sociales, políticos o económicos, competencias que, sin embargo, no podían cuestionarse a Augusto en este momento, arropado ya por el Senado con la mayor parte de sus atribuciones como princeps..$^{38}$

5. Finalmente, el quinto y último elemento del discurso, referido a la revisión y reinterpretación de los Fasti provinciales. En efecto, tomando como referencia los listados de los Fasti Hispanienses de G. Alföldy por provincias, cuyos resultados ratificaba el autor en 2001 "más de treinta años después", ${ }^{39}$ podemos observar lo siguiente:

\footnotetext{
33 AlföLdy 2000a, 61; también AlföLdy 2001, 22: "No es improbable, por lo tanto, que la Transduriana provincia fuera igualmente una "provincia dentro de una provincia", es decir, una región administrativa dentro de la provincia Hispania ulterior, administrada por un legado subordinado al gobernador de dicha gran provincia".

34 Vid. supra n. 28.

35 Estr., III 4.20.

36 JONES 1974, 126.

37 Ibid., 127.

38 Hanslik 1979, cols. 744-754.

39 Alföldy 2001, 26, n. 5.
} 
1. La documentación sobre los presuntos gobernadores de las nuevas provincias es dispar y, en ningún caso, permite establecer coetaneidad para el inicio del período ${ }^{40}$

2. El título de legatus atribuido a los primeros mandatarios de la provincia Lusitania debe ser cuestionado en una fecha anterior a 15 a.C.

3. La existencia de un legatus iuridicus en la Tarraconense (no) implica la vigencia de un nuevo estatuto consular para la provincia, pero no antes del 15-13 a.C. ${ }^{41}$

4. En defecto de un auténtico "primer procónsul" de la Bética (no documentado con seguridad hasta el año 79), ${ }^{42}$ la presencia de un cuestor en la Bética prueba, en cambio, la existencia de la nueva provincia senatorial (ya bajo el gobierno de Augusto), ${ }^{43}$ cuya constitución fue probablemente simultánea con la de Lusitania.

5. Además, la presencia de funcionarios de cierto rango en el séquito de Augusto como el secretario personal de Augusto, desplazado a Segóbriga probablemente durante el tercer viaje de Augusto, entre 15 y 13 a.C., podría indicar que su misión era resolver in situ los problemas planteados, contribuir a la organización del tabularium local con motivo de la promoción de la ciudad como municipium iuris Latini, ${ }^{44}$ o al menos levantar acta detallada de la situación para proponer las soluciones adecuadas después, desde Roma, procedimiento que podía abreviarse contando con la presencia del emperador.

\section{Bibliografía}

Abascal, J. M. (2006): "Los tres viajes de Augusto a Hispania y su relación con la promoción jurídica de ciudades", Iberia 9, 63-78.

Abascal, J. M. - AbAD, L. (eds.) (2003): Las ciudades y los campos de Alicante en época romana, Alicante.

Abascal, J. M. et aliI (2003): Segobriga. Guía del Parque Arqueológico, Madrid.

ALFÖLDY, G.

(1969): Fasti Hispanienses. Senatorische Reichsbeamte und Offiziere in den spanischen Provinzen des Römischen Reiches von Augustus bis Diokletian, Wiesbaden.

(2000): "Das neue Edikt des Augustus aus El Bierzo in Hispanien", ZPE 131, 77-205.

(2000a): Provincia Hispania Superior, Heidelberg.

(2001): "El nuevo edicto de Augusto de El Bierzo en Hispania", [en] L. Grau - J. L. Hoyas (eds.), 2001, 17-27.

(2003): “Administración, urbanización, instituciones, vida pública y orden social”, [en] J. M. Abascal - L. Abad (eds.), 33-57.

Bowman, A. K. et alii (eds.) (1996): The Cambridge Ancient History, vol. X.- The Augustan Empire, 43 BC-69 AD, Cambridge.

BraVo, G.

(2001): Hispania y el Imperio, Madrid.

40 Vid. Alföldy 1969, 303: Hispania Citerior (C. Antistius Vetus: desde 27 a.C.); 307: Lusitania (P. Carisius: desde 26/25 a.C.); 308: Bética (A. Cottius, a comienzos del Imperio).

41 Ibid., 304: L. Calpurnius Piso, documentado para el año 25.

42 Ibid., 308: C. Cornelius Gallicanus.

43 Ibid., 310: L. Licinius Crassus, hacia el 5.

44 Así lo estima Abascal 2006, 72. 
(2007): Hispania. La epopeya de los romanos en la Península, Madrid.

(2011): Nueva historia de la España antigua, Madrid.

Bravo Bosch, M. J. (2008): "La reorganización administrativa de Hispania con César y Augusto", RIDA 55, 107-137.

Crook, J. A. (1996): “Augustus: power, authority, achievement”, [en] A. K. Bowman et alii (eds.), ch. 3, 113-146.

Canali, L. (ed.) (2002): Ottaviano Augusto. Res gestae, Milano.

Costabile, F. - Licandro, O. (2000): Tessera Paemeiobrigensis. Un nuovo editto di Augusto della "Transduriana provincia" e l'imperium proconsulare del princeps, Roma.

EcK, W. (2003): The Age of Augustus, Cornwall.

Fraschetti, A. (1998): Augusto, Roma-Bari.

Galinski, K. (ed.) (2005): The Cambridge Companion to the Age of Augustus, New York.

GonzÁlez Román, C. (ed.) (1999): Ciudades privilegiadas en el occidente romano, Sevilla.

GraU, L. - Hoyas, J. L. (eds.) (2001): El bronce de Bembibre. Un edicto del emperador Augusto del año 15 a.C., Valladolid.

Halfman, H. (1986): Itinera Principum, Geschichte und Typologie der Kaiserreisen im Römischen Reich, Stuttgart.

HansLik, R. (1979): “Augustus”, [en] Kleine Pauly, Bd. 1, München.

Jones, A. H. M. (1974): Augusto, Roma-Bari.

LA RoccA, E. ET ALII (eds.) (2013): Augusto. Catalogo della Mostra, Milano.

Lillo Redonet, F. (2009): “Augusto: la paz de Roma llega a Hispania”, Historia NG 12, 5263.

LóPez BARJA, P. (2000): “La provincia Transduriana”, [en] F. J. Sánchez-Palencia, J. Mangas (coords.), 31-45.

Martín, F. - Gómez pantoja, J. (2001): “El ‘aes bergidense ‘documento singular?”, [en] L. Grau - J. L. Hoyas (eds.), 57-66.

Millar, F. (1966): “The Emperor, the Senate and the Provinces", JRS 56, 155-166.

Navarro Santacana, F. J. (2001): "La presencia del emperador en las ciudades de la Hispania romana" [en] De Augusto a Trajano. Un siglo en la historia de Hispania, Pamplona, 33-55.

Nicolet, C. (1988): L'inventaire du monde. Géographie et politique à Roma aux origines de l'Empire romain, Paris.

PereA, S. (2005): "La batalla de Munda, César y el primer viaje de Octaviano a Hispania, según el testimonio de Nicolás de Damasco", Gerión 23/2, 7-18.

Perea, S. (ed.) (2006): Nicolás de Damasco: Vida de Augusto, Madrid.

Richardson, J. S. (1998): The Romans in Spain, Oxford.

RIESE, A. (1878): Geographi Latini Minores, Hildesheim (reimp. 1964).

Sánchez-Palencia, F. J. - Mangas, J. (coords.) (2000): El Edicto de El Bierzo. Augusto y el Noroeste de Hispania, Madrid.

Salinas, M. (1995): El gobierno de las provincias hispanas durante la República romana (218-27 a.C.), Salamanca.

SANTos, J. (1985): Comunidades indígenas y administración romana en el Noroeste hispánico, Vitoria. 
Syme, R.

(1970): “The Conquest of North-West Spain", [en] Legio VII Gemina, León, 83-107. (1989): La revolución romana, Madrid. 